\title{
Terapia com Células-Tronco em Cardiomiopatia Não-Isquêmica
}

\author{
Ricardo Sanz ${ }^{1}$, Pilar Jiménez-Quevedo ${ }^{1}$
}

\section{RESUMO}

Ao longo da década passada, houve crescente evidência experimental de que os transplantes de células poderiam ser uma abordagem nova e eficiente para a recuperação do miocárdio lesado. Contudo, os estudos pré-clínicos e clínicos sobre terapia celular, em sua maioria, foram feitos em cardiomiopatias isquêmicas. Neste artigo, os autores enfocam a evidência disponível para o tratamento da cardiomiopatia dilatada não-isquêmica com vários tipos de células-tronco.

DESCRITORES: Cardiomiopatia dilatada, terapia. Célulastronco. Terapia tissular. Insuficiência cardíaca congestiva, terapia.

0 coração adulto tem capacidade de regeneração limitada e, portanto, qualquer perda significativa de células, causada por doenças agudas ou crônicas, não tem auto-reparação de forma eficiente, podendo levar ao comprometimento progressivo da função ventricular e, por fim, ao desenvolvimento de insuficiência cardíaca congestiva (ICC).

ICC representa um grave problema de saúde pública, que atinge mais de 5 milhões de norte-americanos $^{1}$. Nos Estados Unidos, a insuficiência cardíaca terminal, com taxas de mortalidade em dois anos de $70 \%$ a $80 \%$, atinge mais de 60 mil pacientes a cada $\mathrm{ano}^{2}$. Apesar de a causa mais significativa de insuficiência cardíaca ser a cardiopatia isquêmica ${ }^{3}$, na prática clínica, a cardiomiopatia dilatada (CMD) é responsável por quase metade dos novos casos de insuficiência cardíaca $^{4-6}$. Os processos cardíacos ou sistêmicos que podem causar CMD ou a ela associados são listados

\footnotetext{
1 Stem Cell Center - Texas Heart Institute at St. Luke's Episcopal Hospital - Houston, Texas - Estados Unidos.

Correspondência: Ricardo Sanz. Stem Cell Center - Texas Heart Institute at St. Luke's Episcopal Hospital - Houston, Texas Estados Unidos. Tel.: 001 (832) 335-8917/001 (713) 355-9704

Fax:001 (832) 355-9440 • E-mail: rsanzruiz@yahoo.es

Recebido em: 19/4/2007 - Aceito em: 2/8/2007
}

\section{SUMMARY}

\section{Stem Cell Therapy in Non-Ischemic Cardiomyopathy}

Over the past decade, there has been accumulating experimental evidence that cell transplantation could be a new, effective approach for repairing damaged myocardium. Nevertheless, most of these preclinical and clinical studies on cell therapy have been performed in ischemic cardiomyopathy. In this paper, the authors focused on the available evidence in the treatment of non-ischemic dilated cardiomyopathy with different types of stem cells.

DESCRIPTORS: Dilated cardiomyopathy, therapy. Stem cells. Tissue therapy. Heart failure, congestive, therapy.

na Tabela 1. Aspectos histológicos comuns a todos os tipos de CMD são perda de cardiomiócitos, hipertrofia das células do miocárdio e uma quantidade variável de fibrose intersticial. A Tabela 2 apresenta algumas das diversas características patológicas dos tipos de CMD não-isquêmica.

Hoje, a terapia para insuficiência cardíaca se restringe ao tratamento de uma moléstia já estabelecida e é, sobretudo, de natureza farmacológica, visando, essencialmente, a inibir o eixo neuro-hormonal que resulta em excessiva ativação cardíaca pelas vias angiotensina ou norepinefrina dependentes. Para pacientes com insuficiência cardíaca em fase terminal, as opções de tratamento são muito limitadas. Transplante cardíaco é a terapia mais eficiente, porém seu uso está comprometido pela limitada obtenção de órgãos doadores (transplantes cardíacos estão disponíveis a menos de 3 mil indivíduos por ano) e por graves problemas médicos e socioeconômicos ${ }^{7,8}$. Outra estratégia possível é o implante de dispositivos de assistência ao ventrículo esquerdo (DAVE); contudo, esse tratamento é caro, está ligado a graves complicações e ainda carece de comprovação a longo prazo ${ }^{9-11}$. Apesar dessas terapias não-farmacológicas e da terapia de ressincronização cardíaca $^{12}$, a insuficiência cardíaca sintomática continua a ter prognóstico sombrio ${ }^{13}$. Assim, tornou-se imperativo o desenvolvimento de novas estratégias te- 
rapêuticas para esses pacientes, dentre as quais a terapia celular tem despertado maior interesse.

Ao longo da década passada, houve crescente evidência experimental de que os transplantes de células poderiam ser abordagem nova e eficiente para a recuperação de miocárdio lesado. Contudo, os estudos pré-clínicos e clínicos sobre terapia celular, em sua maioria, foram feitos em cardiomiopatias isquêmicas. Assim, existem poucos dados pré-clínicos sobre os efeitos do transplante de células no quadro de CMD não-isquêmica, enquanto dados de estudos clínicos são poucos e estudos clínicos randomizados não estão disponíveis. Sob esse aspecto, como na CMD nãoisquêmica, o fluxo coronário epicárdico não fica danificado de forma significativa, e as células enxertadas parecem sobreviver melhor no miocárdio hospedeiro. Dessa forma, ao restaurar a função cardíaca comprometida, o transplante de células poderia trazer novas esperanças para essa doença.

Neste artigo, será abordada a evidência disponível para o tratamento da CMD não-isquêmica com vários tipos de células-tronco.

TABELA 1

Tipos de cardiomiopatia dilatada

\footnotetext{
Cardiomiopatias isquêmicas

Doença valvar: regurgitação mitral, estenose aórtica, regurgitação aórtica

Hipertensão crônica

Taquiarritmias: supraventricular, ventricular, flutter atrial (taquicardia atrial)

Familiar: autossômica dominante, autossômica recessiva - X-ligada

\section{Idiopática}

Toxinas: etanol, antraciclinas, cobalto, agentes antiretrovirais, fenotiazinas, monóxido de carbono, lítio, chumbo, cocaína, mercúrio

Anomalias metabólicas: deficiências nutricionais, distúrbios endócrinos, distúrbios eletrolíticos

Infecciosa: viral, bacteriana, micobacteriana, por espiroquetas, fúngica, parasítica

Distúrbios sistêmicos: lúpus eritematoso sistêmico, artrite reumatóide juvenil, poliarterite nodosa, doença de Kawasaki, distúrbios do colágeno vascular, hematocromatose, amiloidose, sarcoidose

Miocardite por hipersensibilidade

Disfunção peri/pós-parto

Displasia arritmogênica do ventrículo direito

Histiocitose infantil

Distrofias neuromusculares: distrofia muscular de Becker ou de Duchenne

Erros congênitos do metabolismo

Cardiomiopatias mitocondriais

Cardiomiopatia de Keshan

\section{ESTUDOS PRÉ-CLÍNICOS}

\section{Modelos pré-clínicos de cardiomiopatia dilatada}

A CMD em animais pode ser induzida de várias formas e, em sua maioria, os modelos foram realizados em animais pequenos. O primeiro modelo de CMD em ratos foi criado com injeções intraperitoneais de doxorrubicina durante duas semanas ${ }^{14-16}$. O cloridrato de doxorrubicina é uma antraciclina de efeito cardiotóxico, que, um mês após sua administração, induz dilatação da câmara ventricular e insuficiência cardíaca. Com base na evidência de que alguns pacientes apresentam CMD como seqüela de miocardite autoimune prévia, outros pesquisadores induziram miocardite em ratos por meio de imunização com miosina cardíaca de porco ${ }^{17,18}$, resultando em insuficiência cardíaca grave, caracterizada por fibrose cardíaca mais intensa e dilatação ventricular esquerda.

Outros grupos usaram certas linhagens de animais com mutações genéticas que desenvolvem ICC. Esses animais apresentam anomalias congênitas dos músculos cardíacos e esqueléticos, herdadas como traços autossômicos recessivos. Aos primeiros estudos com modelos de distrofia muscular em ratos (dmx) e em cães (distrofia muscular canina ligada ao $\mathrm{X}$ DMCX $)^{19}$ seguiram-se estudos com famílias específicas de hamsters com cardiomiopatia (BIO 14.6, BIO 53.58 e (HF147) $)^{20-22}$.

Esses hamsters desenvolvem CMD caracterizada por câmaras cardíacas dilatadas e paredes ventriculares finas, com miocitólise celular focal.

Vários tipos de células foram testados nos estudos pré-clínicos de CMD, inclusive cardiomiócitos fetais, mioblastos esqueléticos, células mononucleares da medula óssea (CMNMO), células-tronco mesenquimais (CTM) (Figuras 1 e 2) e células endoteliais progenitoras (CEP).

Os primeiros estudos investigaram cardiomiócitos fetais obtidos de embriões ou fetos, que foram direta-

\section{TABELA 2}

Achados característicos de algumas cardiomiopatias dilatadas não-isquêmicas

\section{Cardiomiopatia dilatada idiopática}

Hipertrofia miocitária

Núcleos muito grandes de forma bizarra

Fibrose intersticial

Atrofia miocitária e perda de microfilamentos

Aumento do comprimento da célula sem concomitante aumento de diâmetro

Cardiomiopatia chagásica

Infiltrado mononuclear

Fibrose

Focos da forma leishmaniosa de Trypanosoma cruzi em fibras miocárdicas 


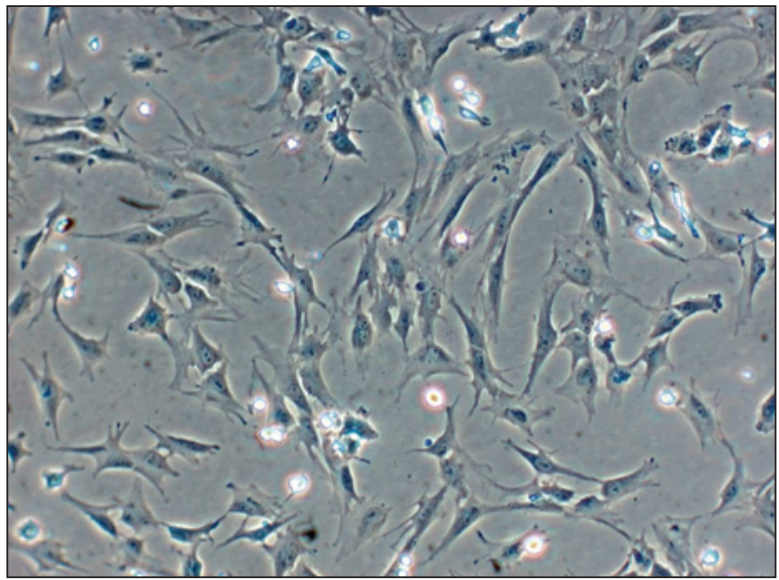

Figura 1 - Cultura primária de células-tronco autólogas mesenquimais da medula óssea (ampliação 25x).

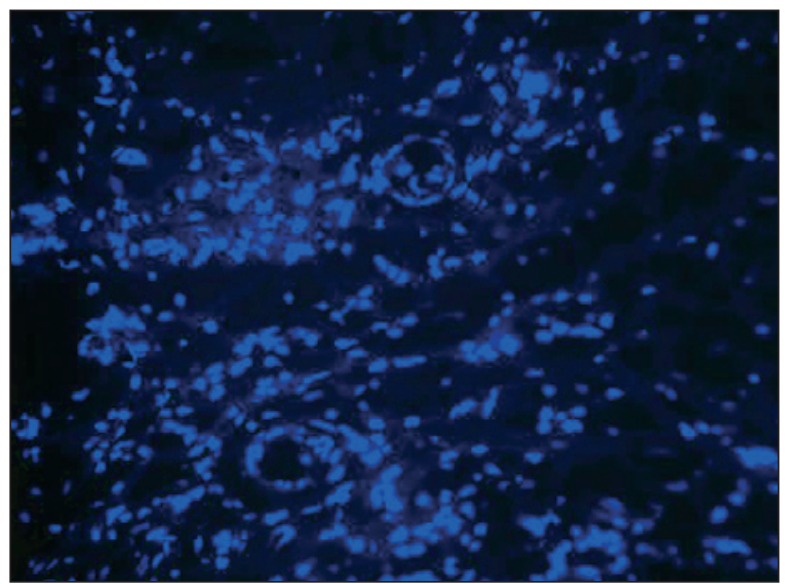

Figura 2 - Marcação DAPI para núcleos de células-tronco mesenquimais alogênicas (ampliação 20x) após injeção transendocárdica em modelo canino de isquemia aguda.

mente injetados no miocárdio ventricular, em animais hospedeiros singênicos, durante cirurgia de coração aberto $^{14,19}$. A seguir, células de músculo liso isoladas do ducto deferente ${ }^{20}$ e células do ventrículo cardíaco do coração de doadores ${ }^{21}$ foram também administradas de maneira semelhante, por toracotomia. Mioblastos esqueléticos foram então estudados quando injetados por via intracoronária ${ }^{15}$ ou durante a cirurgia ${ }^{22}$ e CMNMO foram também usadas $^{16}$ por meio de injeções transepicárdicas no ventrículo esquerdo. Finalmente, a injeção cirúrgica de $\mathrm{CTM}^{17}$ e a injeção intravenosa de $\mathrm{CEP}^{18}$ estão sendo estudadas.

\section{Estudos pré-clínicos}

A terapia com células-tronco para a CMD tem por base a idéia de que o enxerto bem-sucedido das células transplantadas depende da vascularização que supre nutrientes para a sobrevivência. Assim, o miocárdio, com sua circulação coronária epicárdica normal, pode prover um meio melhor para a sobrevivência das células que a cicatriz de um miocárdio infartado.

As primeiras experiências com transplante de células no campo da cardiomiopatia não-isquêmica foram relatadas por Koh et al. ${ }^{19}$, em 1995. Os cardiomiócitos obtidos de fetos caninos foram diretamente injetados no miocárdio ventricular esquerdo de cães singênicos com distrofia muscular, em cirurgia de coração aberto. Os autores provaram que os cardiomiócitos fetais formam enxerto estável no coração de cães adultos, com as células enxertadas justapostas e bem alinhadas aos cardiomiócitos hospedeiros e sem relato de casos de arritmias. Assim, ficou demonstrado que o enxerto celular pode levar a um bem-sucedido aumento do número de cardiomiócitos no coração de cães adultos, porém a competência funcional das células enxertadas não foi avaliada nesse estudo. Outra consideração importante, ao tentar transferir essa abordagem para seres humanos, seria a disponibilidade de células doadoras.

Em 1998, Scorsin et al. ${ }^{14}$ obtiveram cardiomiócitos de fetos e os transplantaram por injeções transepicárdicas no ventrículo esquerdo de ratos singênicos com CMD, relatando, pela primeira vez, melhora no encurtamento fracional por ecocardiografia, apesar de não ter sido estatisticamente significante. Nesse estudo, as células transplantadas não foram detectadas no miocárdio hospedeiro e os autores atribuíram a melhora da contratilidade aos efeitos parácrinos, tais como secreção de fatores de crescimento das células.

Yoo et al. ${ }^{21}$, em 2000, relataram sua experiência pela primeira vez com células cardíacas, ventriculares, pré-marcadas em hamsters com CMD. As células foram obtidas de doadores com cardiomiopatias e injetadas diretamente no miocárdio hospedeiro. Um mês após a cirurgia, as células formaram tecido tipo cardíaco com vasos sanguíneos e sarcômeros. Todavia, o arranjo das células transplantadas era desorganizado, quando comparado àquele do miocárdio hospedeiro. Funcionalmente, foi relatada melhora das pressões sistólicas e desenvolvidas na $\mathrm{dP} / \mathrm{dt}$ e na pressão diastólica nos hamsters transplantados. Em outro estudo, o mesmo grupo de pesquisadores ${ }^{20}$ injetou diretamente células pré-marcadas de músculo liso, tiradas do ducto deferente, na parede anterior do ventrículo esquerdo da mesma linhagem de hamsters. Assim como relatado no estudo anterior, esses autores observaram implante significativo das células injetadas com numerosos capilares no novo tecido muscular, embora as células implantadas apresentassem arranjo desorganizado. Assim, nos dois estudos, os avanços na função diastólica foram atribuídos à maior espessura da parede (que iria limitar a dilatação ventricular), à melhora da função sistólica, à modesta angiogênese percebida na região do transplante celular, e à potencial liberação de fatores de crescimento (não avaliada).

Suzuki et al. ${ }^{15}$, em 2001, infundiram mioblastos esqueléticos via artérias coronárias de coração excisado 
de rato, antes de transplantar os corações novamente para receptores singênicos. Os autores demonstraram que a infusão intracoronária pode, com sucesso, disseminar mioblastos esqueléticos primários em um modelo de rato CMD, que essas células se diferenciam em miotubos multinucleados alinhados com o eixo da fibra cardíaca, e que esse procedimento está associado a melhora significativa da função cardíaca $(\mathrm{dP} / \mathrm{dt}$ aumentada e melhores curvas pressão/volume). Não foram relatados casos de embolias coronárias. Os mecanismos propostos incluíam propriedades dos miotubos enxertados, liberação de fatores parácrinos, melhor angiogênese e melhor capacidade contrátil dos cardiomiócitos.

Os próximos experimentos com terapia celular em cardiomiopatias não-isquêmicas foram feitos por Ishida et al. ${ }^{16}$, em 2004. Esses autores injetaram CMNMO não-marcadas em ratos, por toracotomia, demonstrando os efeitos benéficos, sobretudo para a função sistólica. O transplante de células também evitou remodelamento ventricular, atenuando mudanças causadas por doxorrubicina, e aumentou a densidade do vaso sanguíneo. Para explicar seus achados, os autores consideraram a angiogênese, que abrangia o coração inteiro, como principal mecanismo.

Pouly et al. ${ }^{22}$, em 2004, pesquisaram mioblastos esqueléticos autólogos introduzidos em hamsters por meio de múltiplas injeções intramiocárdicas diretas, e encontraram um implante consistente das células, sem aumento da quantidade de fibrose intersticial e evitando a função ventricular esquerda como avaliado in vivo por ecocardiografia. Algumas das possíveis explicações mecânicas sugeridas foram mudanças na matriz extracelular, contração dos miotubos e secreção de fatores de crescimento. Todavia, o tempo de acompanhamento foi curto (um mês) e não foi feita avaliação do risco pró-arrítmico.

Por fim, em 2005, o fulcro de interesse foram as CTM e as CEP. Primeiro, Nagaya et al. ${ }^{17}$ injetaram cirurgicamente CTM obtidas da medula óssea em ratos, demonstrando os seguintes efeitos: 1) indução de miogênese e angiogênese; 2) diferenciação das CTM em cardiomiócitos, células endoteliais vasculares e células de músculo liso; 3) secreção de grandes quantidades de fator de crescimento endotelial vascular (FCEV), fator de crescimento de hepatócitos (FCH), adrenomedulina e fator de crescimento tipo insulina1 (FCl-1); 4) melhora da função cardíaca e inibição do remodelamento ventricular; e 5) redução do volume de fração de colágeno no miocárdio. Com todos esses dados, os autores concluíram que as CTM melhoram a função cardíaca na CMD, possivelmente por meio da indução da miogênese e da angiogênese, bem como pela inibição da fibrose miocárdica. Por outro lado, em estudo recente realizado por Werner et al. ${ }^{18}$, CEP derivadas do baço, Dil-marcadas e de doadores sadios foram introduzidas por via intravenosa, procedi- mento repetido durante duas semanas consecutivas, em um modelo com miocardite de CMD em ratos. Os principais achados desse estudo foram que as CEP atenuam o dano da miocardite, melhoram a função ventricular esquerda à ecocardiografia e a espessura da parede à histopatologia, e reduzem o teor de tecido fibroso. É interessante que a transformação das CEP em células endoteliais residentes e maduras (intensificação da rede capilar) poderia ser apenas um dos possíveis mecanismos desse benefício.

\section{EXPERIÊNCIAS CLÍNICAS}

No campo da terapia celular na CMD não-isquêmica, ainda são necessários experimentos randomizados. Os experimentos com seres humanos ainda não foram publicados e se limitam a comunicações pessoais em conferências recentes. Dois grupos relataram pequenas séries de pacientes com CMD não-isquêmica tratados com células-tronco nas últimas Sessões Científicas do American College of Cardiology (Atlanta, 2006) ${ }^{23,24}$. Nesses estudos, o transplante de CMNMO por via intracoronária ou por injeção diretamente no miocárdio parecia seguro e factível, com moderada melhora tanto clínica como funcional. Contudo, os resultados desses estudos não-randomizados com amostras pequenas $(n=8$ e $n=6)$ devem ser considerados com cautela.

Há alguns experimentos relatados sobre terapia com células-tronco em tipos específicos de CMD, como, por exemplo, na cardiomiopatia chagásica. A doença de Chagas, doença infecciosa causada pelo Trypanosoma cruzi e que leva à dilatação do ventrículo esquerdo e FCH (Tabela 2), ainda é endêmica na América Central e na América do Sul, atingindo 11 milhões de pessoas. A esse respeito, Vilas-Boas et al. ${ }^{25}$ relataram pela primeira vez o caso de um homem de 52 anos com CMD chagásica tratada por infusão intracoronária de CMNMO. Por meio de ventriculografia por radionuclídeos, esses autores observaram aumento de $24 \%$ a $32 \%$ da fração de ejeção do ventrículo esquerdo e de $28 \%$ a $38 \%$ por ecocardiografia após um mês. Os autores também relataram melhora significativa da classe funcional da New York Heart Association (NYHA) no teste de marcha de 6 minutos e na qualidade de vida. É digno de nota que não foram observados eventos adversos.

\section{CONCLUSÕES}

Os dados sobre transplantes em CMD não-isquêmica são escassos, porém animadores. Nesse campo, a base lógica para terapia celular é restaurar cardiomiócitos em um ambiente com melhor irrigação que aquele observado nos tecidos com infarto do miocárdio, melhorando assim a função cardíaca e o prognóstico de pacientes com ICC.

Até agora, os estudos pré-clínicos confirmaram que o enxerto de células-tronco de diferentes tipos no mio- 
cárdio melhora a perfusão deste e a função cardíaca. Contudo, ainda no terreno das possibilidades pré-clínicas, a evidência disponível nos modelos pré-clínicos de CMD irá, inevitavelmente, nos conduzir para o ambiente clínico. Esses primeiros estudos, além de apresentar resultados promissores, levantam as mesmas perguntas que são feitas em relação à cardiomiopatia isquêmica e que precisam ser respondidas, para que futuros experimentos possam ser desenhados com a metodologia e os objetivos apropriados.

Por causa da natureza da doença, a administração das células deveria idealmente ser feita com técnicas baseadas no cateter, permitindo distribuição disseminada das células de forma minimamente invasiva. CTM e CEP parecem ser os tipos mais promissores de células, com seus benéficos efeitos miogênicos e angiogênicos, apesar de que mioblastos esqueléticos e CMNMO poderiam ter um papel a desempenhar. Pacientes com CMD que não são candidatos adequados para transplante ou dispositivos mecânicos de assistência e com estágios avançados de ICC seriam os candidatos ideais para essa terapia.

\section{REFERÊNCIAS BIBLIOGRÁFICAS}

1. Cohn JN, Bristow MR, Chien KR, Colucci WS, Frazier $\mathrm{OH}$, Leinwand LA, et al. Report of the National Heart, Lung, and Blood Institute Special Emphasis Panel on Heart Failure Research. Circulation. 1997;95(4):766-70.

2. The CONSENSUS Trial Study Group. Effects of enalapril on mortality in severe congestive heart failure. Results of the Cooperative North Scandinavian Enalapril Survival Study (CONSENSUS). N Engl J Med. 1987;316(23):1429-35.

3. Mahon NG, O'Rorke C, Codd MB, McCann HA, McGarry K, Sugrue DD. Hospital mortality of acute myocardial infarction in the thrombolytic era. Heart. 1999;81(5):478-82.

4. Codd MB, Sugrue DD, Gersh BJ, Melton LJ $3^{\text {rd }}$. Epidemiology of idiopathic dilated and hypertrophic cardiomyopathy: a population-based study in Olmsted Country, Minnesota, 1975-1984. Circulation. 1989;80(3):564-72.

5. Dec GW, Fuster V. Idiopathic dilated cardiomyopathy. N Engl J Med. 1994;331(23):1564-75.

6. Packer M, Bristow MR, Cohn JN, Colucci WS, Fowler MB, Gilbert EM, et al. The effect of carvedilol on morbidity and mortality in patients with chronic heart failure. $N$ Engl J Med. 1996;334(21):1349-55.

7. Hogness JR. The artificial heart: prototypes, policies and patients. Washington:National Academy Press;1991.

8. Annual Report of the US Scientific Registry for Organ Transplantation and the Organ Procurement and Transplantation Network - 1990. Washington: US Department of Health and Human Services;1990.

9. Frazier $\mathrm{OH}$, Rose EA, Macmanus Q, Burton NA, Lefrak EA, Poirier VL, et al. Multicenter clinical evaluation of the HeartMate 1000 IP left ventricular assist device. Ann Thorac Surg. 1992;53(6):1080-90.

10. McCarthy PM, Portner PM, Tobler HG, Starnes VA, Ramasamy $N$, Oyer PE. Clinical experience with the Novacor ventricular assist system. Bridge to transplantation and the transition to permanent application. J Thorac Cardiovasc Surg. 1991;102(4):578-87.

11. Oz MC, Argenziano M, Catanese KA, Gardocki MT, Goldstein DJ, Ashton RC, et al. Bridge experience with long-term implantable left ventricular assist devices. Are they an alternative to transplantation? Circulation. 1997;95(7):1844-52.

12. Konstam MA. Progress in heart failure management? Lessons from the real world. Circulation. 2000;102(10):1076-8.

13. Young JB, Abraham WT, Smith AL, Leon AR, Lieberman $R$, Wilkoff $B$, et al. Combined cardiac resynchronization and implantable cardioversion defibrillation in advanced chronic heart failure: the MIRACLE ICD Trial. JAMA. 2003;289(20): 2685-94.

14. Scorsin M, Hagege AA, Dolizy I, Marotte F, Mirochnik N, Copin $\mathrm{H}$, et al. Can cellular transplantation improve function in doxorubicin-induced heart failure? Circulation. 1998; 98(19 suppl II):II-151-5.

15. Suzuki K, Murtuza B, Suzuki N, Smolenski RT, Yacoub MH. Intracoronary infusion of skeletal myoblasts improves cardiac function in doxorubicin-induced heart failure. Circulation. 2001;104(12 suppl I):I-213-7.

16. Ishida $M$, Tomita $S$, Nakatani $T$, Fukuhara $S$, Hamamoto $M$, Nagaya $\mathrm{N}$, et al. Bone marrow mononuclear cell transplantation had beneficial effects on doxorubicin-induced cardiomyopathy. J Heart Lung Transplant. 2004;23(4):436-45.

17. Nagaya N, Kangawa K, Itoh T, Iwase T, Murakami S, Miyahara $\mathrm{Y}$, et al. Transplantation of mesenchymal stem cells improves cardiac function in a rat model of dilated cardiomyopathy. Circulation. 2005;112(8):1128-35.

18. Werner L, Deutsch V, Barshack I, Miller H, Keren G, George J. Transfer of endothelial progenitor cells improves myocardial performance in rats with dilated cardiomyopathy induced following experimental myocarditis. J Mol Cell Cardiol. 2005;39(4):691-7.

19. Koh GY, Soonpaa MH, Klug MG, Pride HP, Cooper BJ, Zipes DP, et al. Stable fetal cardiomyocyte grafts in the hearts of dystrophic mice and dogs. J Clin Invest. 1995;96(4):2034-42.

20. Yoo KJ, Li RK, Weisel RD, Mickle DA, Li G, Yau TM. Autologous smooth muscle cell transplantation improved heart function in dilated cardiomyopathy. Ann Thorac Surg. 2000;70(3):859-65.

21. Yoo KJ, Li RK, Weisel RD, Mickle DA, Jia ZQ, Kim EJ, et al. Heart cell transplantation improves heart function in dilated cardiomyopathic hamsters. Circulation. 2000;102(19 suppl III):III-204-9.

22. Pouly J, Hagege AA, Vilquin JT, Bissery A, Rouche A, Bruneval $P$, et al. Does the functional efficacy of skeletal myoblast transplantation extend to nonischemic cardiomyopathy? Circulation. 2004;110(12):1626-31.

23. Trainini JC, Lago NE, Alvarez M, Christen Al, Guevara E, Mouras JH, et al. Mononuclear bone marrow stem cells implant as an alternative treatment in non-ischemic dilated cardiomyopathy. American College of Cardiology Scientific Sessions, Atlanta 2006 (personal communication).

24. Kalil RA, Ott DR, Sant'Anna RT, Dias EM, Bordignon S, Nardi NB, et al. Intramyocardial bone marrow stem cell transplantation for non-ischemic dilated cardiomyopathy: four month follow-up. American College of Cardiology Scientific Sessions, Atlanta 2006 (personal communication).

25. Vilas-Boas F, Feitosa GS, Soares MB, Pinho-Filho JA, Mota A, Almeida AJ, et al. Bone marrow cell transplantation to the myocardium of a patient with heart failure due to Chagas' Disease. Arq Bras Cardiol. 2004;82:185-7. 